\title{
Neuromodulation with transcranial focused ultrasound
}

\author{
Jan Kubanek, PhD \\ Departments of Neurobiology and Radiology, Stanford University School of Medicine, Stanford, California
}

The understanding of brain function and the capacity to treat neurological and psychiatric disorders rest on the ability to intervene in neuronal activity in specific brain circuits. Current methods of neuromodulation incur a tradeoff between spatial focus and the level of invasiveness. Transcranial focused ultrasound (FUS) is emerging as a neuromodulation approach that combines noninvasiveness with focus that can be relatively sharp even in regions deep in the brain. This may enable studies of the causal role of specific brain regions in specific behaviors and behavioral disorders. In addition to causal brain mapping, the spatial focus of FUS opens new avenues for treatments of neurological and psychiatric conditions. This review introduces existing and emerging FUS applications in neuromodulation, discusses the mechanisms of FUS effects on cellular excitability, considers the effects of specific stimulation parameters, and lays out the directions for future work.

https://thejns.org/doi/abs/10.3171/2017.11.FOCUS17621

KEY WORDS noninvasive; neurointervention; stimulation; deep brain; brain mapping; neuroplasticity

$\mathrm{O}$ UR ability to control or influence neural activity in the human brain has rested on methods that are either invasive or limited by a broad radius of action. Deep brain stimulation (DBS) involves a surgical procedure in which leads carrying stimulation electrodes are implanted into specific nuclei deep within the brain. Electric currents of certain pulsing protocols make it possible to increase, suppress, or distort neuronal activity in the circuits near a particular electrode..$^{5}$ This approach has been successfully used to alleviate the symptoms associated with medication-refractory action or resting tremor in patients with essential tremor (ET) or Parkinson's disease. ${ }^{14,44,46}$ The main strength of DBS is that it provides stimulation throughout the lifetime of the implanted device, which generally results in lasting treatments. The main drawback of DBS is that the associated surgeries may lead to complications such as infection or hemorrhage. ${ }^{3,4}$

Researchers and clinicians have been considering alternative, noninvasive approaches to neurostimulation. In particular, transcranial magnetic stimulation (TMS) and transcranial current stimulation (TCS) can facilitate or inhibit neural activity through the intact skull and skin. Using TMS, a brief pulse or a burst of magnetic energy applied to motor cortex can evoke instantaneous movement. ${ }^{45}$ This approach can be used to alter or probe the function of corticospinal pathways. ${ }^{37}$ Moreover, TMS application in hour-long sessions over multiple days can improve the condition of patients suffering from major depressive disorder. ${ }^{28,36}$ TCS can also alter corticospinal excitability, but unlike TMS, it does not elicit action potentials..$^{23,43}$ The main prospects for TCS lie in its repeated application using specific stimulation protocols. This is a topic of active investigation. Both TMS and TCS suffer from a common drawback - a broad radius of action (on the order of centimeters). This limitation is particularly pronounced in deep brain applications. The spatial constraint derives from the fact-captured by Maxwell's equations - that purely magnetic or electric fields cannot attain a maximum outside of their sources. To improve focus at depth, researchers have attempted to superimpose magnetic or electric fields by using multiple sources. ${ }^{7,20}$ However, a tightening of focus in a central region accompanies a deterioration in regions surrounding the ancillary sources. Thus, noninvasive and targeted stimulation of specific nuclei or pathways deep in the brain has remained elusive.

\section{Transcranial Focused Ultrasound}

Transcranial focused ultrasound (FUS) is a novel neuromodulation approach that combines noninvasiveness and sharp spatial focus. ${ }^{12,41}$ As its basis, ultrasound comprises a pressure wave of frequencies above the audible

ABBREVIATIONS ARF = acoustic radiation force; DBS = deep brain stimulation; ECoG = electrocorticography; $E T=$ essential tremor; FUS = focused ultrasound; TCS = transcranial current stimulation; TMS = transcranial magnetic stimulation. 
range. As a propagating wave, ultrasound can penetrate biological tissues including the skull, and its energy can be concentrated into a small, circumscribed region. The diameter of the stimulated volume is typically several millimeters for applications through the human skull, ${ }^{18}$ and can attain approximately $100 \mu \mathrm{m}$ in soft-tissue applications. ${ }^{39}$ FUS can excite or inhibit cellular activity, depending on specific stimulation parameters ${ }^{47}$ FUS can cause a transient increase in firing rates in motor cortex and in the retina with short latency, ${ }^{39,55}$ and thus has a direct capability to influence cellular discharge. It has been hypothesized that these effects are mediated by ion channels that can detect changes in membrane stretch following a propagating pressure wave. ${ }^{30,31,49,57,58}$ Other mechanisms may also be at play; this topic is discussed in more detail below. Besides transient effects, FUS applied for a substantial time period (> 10 seconds) can induce long-term changes in neural activity of the stimulated circuits. ${ }^{8,59}$ The mechanisms underlying such lasting effects are not understood. In addition to its intrinsic neurostimulatory potential, the effects of FUS can be enhanced or further controlled using nanoparticles that contain a specific neuromodulatory drug of interest. Upon FUS impact, the nanoparticles circulating in the bloodstream release their drug load specifically at the site of the FUS target. ${ }^{1}$ Using propofol as the neuroactive substance, one can, for instance, strongly inhibit the activity in specific brain circuits in a relatively noninvasive manner.

The combination of noninvasiveness, depth penetration, and spatial focus of neuromodulatory FUS can be harnessed in a variety of applications. The transient and long-term modes of operation invite applications in causal brain mapping and treatments based on neuroplasticity, respectively. To study the causal involvement of a brain region in a behavior or a behavioral disorder, a researcher or a clinician delivers a brief, low-intensity stimulation pulse into the region of interest, and measures the effect on the behavior of interest. Until now, such causal inference could only be performed in cortical regions using TMS and at a low spatial resolution. FUS opens the possibility of probing the role of deep brain regions as well and at a relatively high spatial resolution. Besides brain mapping, FUS may also enable noninvasive treatments, harnessing the neuroplastic effects of the modality. If FUS is applied for periods of $>10$ seconds, it can induce long-term changes in the neural activity of target circuits. ${ }^{8,59}$ This mode of operation has the potential for disrupting aberrant patterns of neural activity in a lasting manner. This may enable noninvasive treatments of brain disorders.

Focused ultrasound may provide a unique tool to identify the brain regions involved in disorders such as ET, neuropathic pain, and possibly also depression. Let us consider an example use of FUS in the diagnosis and treatment of the neural circuits involved in ET. A clinician aims to determine, in a given patient with tremor, which brain regions are most strongly implicated in generating the signs (Fig. 1). The patient's head is enclosed in a readily available array of ultrasound transducers. ${ }^{18}$ The patient is advanced into an MR scanner to enable a visualization of the FUS target. The tremor amplitude is continuously recorded using a data glove. The operator sets

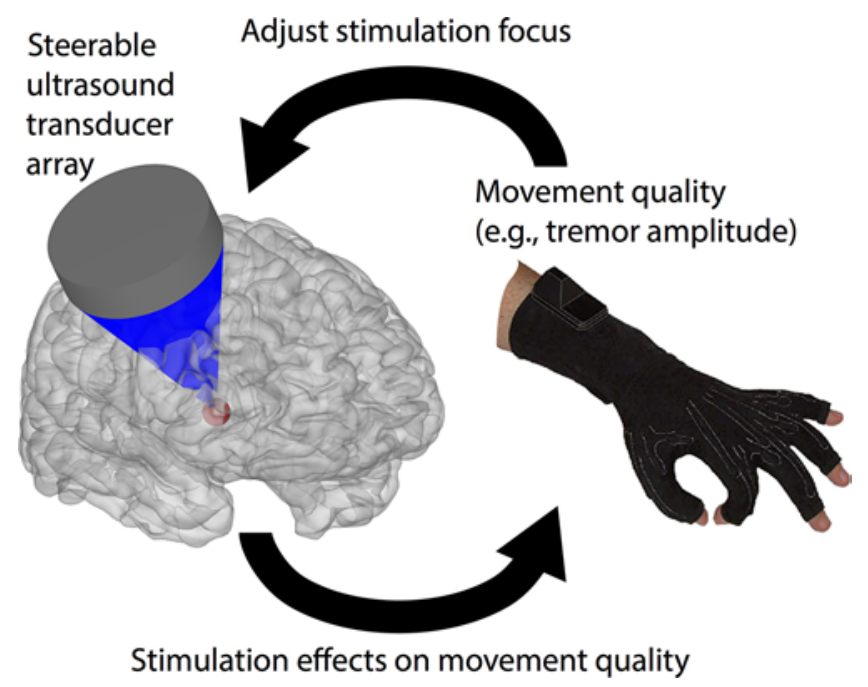

FIG. 1. Illustration of the application of transcranial FUS to determine the brain regions involved in specific behaviors and behavioral disorders.

the coordinates of the FUS target, delivers a neuromodulatory pulse, and measures the concomitant reduction in the tremor amplitude. The region that reduces the tremor amplitude most strongly becomes the target of follow-up treatment.

The treatment can be mediated using the same FUS system, in two ways. First, long-term FUS stimulation of the target may induce lasting changes in the stimulated circuits, ${ }^{8}$ which may help to alleviate certain signs. Second, it is possible to increase the stimulation intensity by $>$ 2 orders of magnitude and in this way to ablate the target..$^{18}$ This nonsurgical and nonionizing intervention is specific to the FUS target; neighboring regions remain intact. Treatment can also be mediated using the invasive DBS, but now in a targeted manner: the DBS leads are placed into the region identified by FUS to underlie the signs in the given patient.

A similar procedure might enable us to identify the neural circuits involved in neuropathic pain. This application, however, relies on subjective reports of immediate changes in pain perception. Such subjective reports may be unreliable and may thus misguide the brain mapping procedure. This issue is even more pronounced in the hypothetical identification of the brain regions involved in depression. Thus, it is anticipated that FUS-based brain mapping will find its main uses in establishing the neural circuits associated with disorders in which the criterion to be optimized rests on an objective metric, as is the case with movement disorders.

On the basic science side, FUS has the potential to become a spatially focused alternative to TMS in cognitive neuroscience and psychology. In particular, FUS is the only noninvasive modality that can be used to stimulate, with sufficiently tight focus, specific circuits deep in the brain. In this regard, circuits that constitute the striato-pallido-thalamic network are of particular interest because of their involvement in the generation of movements and in the assessment of values associated with decision options. Establishing the exact role of the individual nuclei 
and pathways in the respective functions in humans would present a major step forward in enabling treatments of the associated brain disorders.

The potential of FUS to modulate cellular excitability has been known for almost 100 years. ${ }^{13,17,22,34}$ The current surge of interest in it as a noninvasive neurostimulation modality has been triggered by the following relatively recent findings: 1) FUS can elicit neuromodulatory effects in the CNS by using relatively short stimuli; $; 9$ 2) short stimuli of low intensity can trigger visible movements upon motor cortex stimulation in rodents; ${ }^{25-27,35,38,56,62}$ and 3) the method has been safely used in primates, including humans. ${ }^{9,21,32-34,60}$ Given the enormous potential of this neurostimulation modality in causal brain mapping and treatments of deep brain circuits, work is currently under way to provide the information necessary for effective use. In particular, researchers are beginning to elucidate how FUS stimulates neurons and which FUS parameters mediate effective excitation or inhibition. Important future points of inquiry include investigations of the capability of FUS to induce plastic changes in the stimulated circuitry and studies of the associated safety limits.

\section{Mechanism of Ultrasonic Neuromodulation}

The knowledge of how FUS mediates cellular discharge could greatly facilitate the determination of effective stimulation parameters. At a high level, FUS can act on target tissue by using two main mechanisms-it can heat up the target (thermal effect), or it can physically distort or displace the target (mechanical effect). Recent ultrasound neurostimulation studies have been typically performed at relatively low pressures ( $<0.6 \mathrm{MPa}$ at focus), low frequencies (sub-MHz), and short pulse durations $(\leq 300 \mathrm{msec}$ ). These protocols have led to temperature increases of $\leq 0.01^{\circ} \mathrm{C} . .^{12,41}$ Thus, when using common neuromodulation protocols, heating is not considered to be a main driver of the effects. A recent study ${ }^{31}$ using Caenorhabditis elegans specifically tested whether the FUS neuromodulatory effects can be of a thermal or mechanical nature. The animals rapidly reversed direction upon FUS impact. It was found that animals deficient in thermosensation responded to FUS just like wild types, whereas animals incapable of sensing tiny mechanical forces failed to respond to FUS. This suggests that the effects of FUS on excitable cells have a strong mechanical basis.

Regarding the molecular mechanism, it has long been known that FUS stimulation of human fingers or arms can lead to tactile or nociceptive sensations, as well as sensations of warmth and cold.$^{15}$ These experiments have provided evidence that FUS can activate certain classes of molecular receptors in the skin. This has fueled the hypothesis that the pressure wave associated with propagating FUS might lead to membrane stretch and thus to an opening of certain classes of ion channels, such as mechanosensitive ion channels. ${ }^{30,57,58}$ This hypothesis has recently been confirmed using $C$. elegans. It has been found that mutants that lack a pore-forming subunit of an ion channel that is critical for sensing gentle touch show a profound deficit in the responsiveness to FUS. ${ }^{31}$ Thus, a mechanosensitive ion channel is required for the FUS re- sponse in this animal. Patch-clamp recordings in another recent study further revealed FUS effects on ion channels of the Piezo family. ${ }^{49}$ These channels are also known to participate in mechanosensation ${ }^{53}$ and are expressed in the brain. ${ }^{61}$ These findings suggest that the mechanical forces associated with propagating FUS are of sufficient magnitude to induce membrane displacement and so mediate a conformational change of ion channels embedded within. These studies illuminate one complete pathway of the FUS action, which initiates with the forces associated with the propagating ultrasound and terminates with an activation of ion channels sensitive to membrane tension. In this regard, it is worth noting that mechanosensation plays a central role in the neurogenesis, maintenance, and repair of the CNS. ${ }^{57}$ If FUS can tap into this signaling, it may evolve into a novel tool for noninvasive neuroregeneration.

Ultrasound can exert several possible forms of mechanical forces on cellular membranes. First, ultrasound may elicit cavitation, a phenomenon characterized by formation and collapse of gaseous bodies in liquid media or soft tissues. Experimentally determined cavitation thresholds in soft tissues require an in situ pressure of $\geq 1.9 \mathrm{MPa} .{ }^{42}$ The thresholds increase with frequency, and are much higher in the brain. In comparison, the majority of recent neuromodulation studies have used peak pressures $<0.6$ $\mathrm{MPa}$ in the brain. ${ }^{6}$ Thus, cavitation requires much higher pressures than those currently used for neuromodulation. Second, the target tissue, such as a cell membrane, experiences oscillations with period equal to the ultrasound carrier frequency. The pressures used for neuromodulation can cause appreciable particle displacement (on the order of $\left.0.01-0.1 \mu \mathrm{m}^{16}\right)$. Nonetheless, the displacement is distributed in sinusoidal fashion along the wavelength of the propagating wave. This creates a very small displacement gradient (e.g., $0.1 \mu \mathrm{m}$ per $100 \mu \mathrm{m}^{31}$ ). It is questionable whether such a small gradient can cause significant enough deformation of a pore segment of an ion channel with regard to the channel dimensions. The third and most probable form of mechanical energy is the acoustic radiation force (ARF) ${ }^{24,52,54}$ The $\mathrm{ARF}$ is associated with momentum transfer from the ultrasound wave field to the medium $;{ }^{10}$ the ARF exerts a steady pressure on a target throughout the time of ultrasound application. This steady pressure may stretch a cell membrane to an extent that affects conformation states of ion channels or other active molecules tied to the membrane..$^{31}$

In addition to direct effects on ion channels, it has been hypothesized that the oscillating FUS pressure wave might lead to oscillations in the membrane capacitance. Changes in capacitance may in turn lead to changes in the membrane potential. This hypothesis has not, thus far, found robust experimental support. ${ }^{50,51}$ A relatively recent model based on this idea, "intramembrane cavitation," $29,47,48$ predicts a profound drop of the membrane potential in response to FUS onset. The proposed drop measures $\geq 100 \mathrm{mV}$ in the hyperpolarizing direction and can be observed for a period of several milliseconds. Thus far, such a marked drop in membrane voltage in response to FUS has not been confirmed in direct cellular recordings. ${ }^{30,50,58}$ 


\section{Effective Stimulation Protocols}

An ultrasound stimulus is defined by five main parameters-carrier frequency, peak intensity, duration, pulse repetition frequency, and duty cycle. Each of these parameters can have a strong effect on the stimulation outcome.

The carrier frequency is a crucial parameter that defines the spatial extent of the FUS focus and the quality of the FUS transmission through the skull. Higher frequencies are associated with shorter wavelengths and so can attain a relatively sharper spatial focus. On the other hand, higher frequencies propagate through the skull less effectively compared with lower frequencies. ${ }^{6}$ For animals with a thin skull such as mice, it is possible to use frequencies as high as $5 \mathrm{MHz}$ and still penetrate the skull. ${ }^{35}$ This enables a focal width as small as $0.8 \mathrm{~mm} .{ }^{35}$ For applications in humans, in which the skull presents a significant barrier to the propagating ultrasound, carrier frequencies between $250 \mathrm{kHz}$ and $500 \mathrm{kHz}$ have commonly been used. ${ }^{32-34}$ In this frequency range, the focal width is on the order of several millimeters.

Skull transmission aside, the carrier frequency influences the effectiveness of the actual neuronal stimulation. In retinal cells, higher carrier frequencies require lower FUS pressures to induce cellular discharge activity. ${ }^{40}$ The effect has been faithfully captured using a model that rests on the ARF, based on the fact that ARF increases roughly linearly (proportional to $[\sim] f^{1.18}$ ) with increasing carrier frequency $(f) .{ }^{19}$ Thus, if ARF is the form of mechanical energy that drives the neurostimulatory effects, higher FUS frequencies should lead to stronger effects on cellular excitability. ${ }^{40}$ In contrast, the probability of observing behavioral responses to FUS stimulation of motor regions in mice is higher at lower FUS frequencies. ${ }^{27,62}$ The frequency-dependence mismatch between these data and those obtained in the retina may be explained by the increase in focal width with decreasing frequencies. ${ }^{40}$ In a diffraction-limited system, lower frequencies activate a much larger area $\left(\sim 1 / f^{2}\right)$ and volume (up to $\left.\sim 1 / f^{3}\right)$. Stimulation of a larger volume of the brain increases the probability of activation of neurons involved in the behavioral responses. The seeming discrepancy between these two results underscores the necessity to keep the extent of the stimulated tissue constant when studying the neurostimulatory effects of the carrier frequency.

In regard to FUS intensity-which is proportional to FUS pressure squared-stronger stimuli generally lead to more pronounced effects. ${ }^{12,41}$ However, distinct levels of stimulus intensity may entail qualitatively distinct mechanisms (e.g., mechanical for low-intensity and thermal for high-intensity stimuli). In this way, stimulus intensity may govern distinct natures of the elicited effects. For example, a study investigated the effects of FUS intensity and duration on electrocorticographic $(\mathrm{ECoG})$ signals recorded from cat cortex. Low-intensity stimuli generally had an excitatory effect on the ECoG rhythms, whereas stimuli of higher intensity suppressed the rhythms. ${ }^{59}$

The likelihood of observing behavioral responses such as limb movements in rodents or reversals in $C$. elegans increases with increasing FUS duration; the effects begin to saturate for stimuli of approximately $100 \mathrm{msec}$ in duration. ${ }^{27,31}$ In this regard, interestingly, recent neuromodu- lation studies in large mammals including humans used stimuli that were applied for $\geq 100$ msec. . $^{932-34,60}$

A recent study conducted a meta-analysis of multiple reports of excitation and inhibition. ${ }^{47}$ The study suggested that FUS intensity dictates whether a net outcome is an excitation or an inhibition as much as does a specific FUS pulsing protocol. In particular, short, repetitive pulses of the FUS-which correspond to low values of the duty cycle-are more likely to produce an inhibition, whereas longer repetitive pulses-higher duty cycle values-are more likely to lead to an excitation. This dependence reflects the proposition that the activity of certain classes of ion channels can be sensitive to the duty cycle. ${ }^{47}$ For example, low-threshold spiking interneurons expressing T-type calcium channels may be activated using pulses of short duty cycles, which may lead to a net inhibition. ${ }^{47}$

The peculiarities associated with such models underscore the necessity to gain a firm grasp on the detailed mechanisms of the FUS action. For example, if the main mechanism of FUS action rests on an activation of specific classes of ion channels, then whether one can expect excitatory or inhibitory net effects depends on the type of the channels in a given tissue, on their expression level, and also on their potentially unique sensitivity to specific FUS parameters. ${ }^{31}$ Thus, whether a specific set of FUS parameters leads to an excitation or an inhibition may also depend on tissue properties.

\section{Neuroplastic Potential}

FUS may find applications in inducing plastic, lasting effects in the stimulated circuitry. Recent stimulation protocols have typically aimed at acute effects that accompany relatively brief FUS stimuli. However, it has been shown that FUS applied for prolonged periods of time can lead to lasting effects on neuronal activity, with the effects being commonly suppressive. For example, amplitudemodulated FUS (modulation frequency of $\geq 8 \mathrm{~Hz}$ ) applied for $\geq 30$ seconds led to a long-term reduction of the amplitude of ECoG rhythms by $30 \%-40 \% .{ }^{59}$ As another example, a recent 40-second sonication protocol reduced the amplitude of somatosensory evoked potentials recorded in pig cortex by approximately 30\%.8 The effect was longlived, lasting $\geq 10$ minutes. The reports of long-term yet reversible effects open the possibility that FUS could be used to treat brain disorders through long-term effects on neural activity or on the state of the stimulated neural circuitry. This research direction remains to be explored.

\section{Safety and Practicality}

The FDA safety limits for ultrasound exposure have been based on diagnostic ultrasound. ${ }^{2}$ Diagnostic ultrasound rests on brief pulses of high-frequency (commonly $\geq 5 \mathrm{MHz}$ ) stimuli. In comparison, neuromodulatory ultrasound typically comprises longer pulses at low carrier frequencies (sub-MHz range). Both the pulse duration and carrier frequency can be accommodated by current FDA guidelines-by using the time-average intensity and the mechanical index, respectively, as the limiting metrics. However, whether these exposure limits provide adequate guidelines for long-term neuromodulatory FUS-which 
appears to be capable of triggering plastic changes in neural circuits ${ }^{26}$-remains to be validated.

Finally, it is worthwhile to consider the practicality of the FUS approach. Currently, patients undergoing highintensity, ablative FUS treatments have their hair shaved prior to the main procedure. ${ }^{11}$ This step is taken mainly due to concerns that high-intensity FUS might result in a significant thermal rise on the hair surface or within the skin. Low-intensity, neuromodulatory FUS does not incur such risks, and so it is expected that its future applications will not require hair shaving. This is an important premise especially for future FUS uses in cognitive neuroscience.

\section{Conclusions}

In summary, FUS is the only neuromodulation modality to date that combines noninvasiveness, depth penetration, and spatial focus. Short bursts of low-intensity FUS can stimulate or inhibit neurons and other excitable cells. Current efforts are being directed at determining which FUS parameters maximize cellular excitation and inhibition. These efforts are being supported by ongoing work that investigates the exact mechanism of the FUS action. With this knowledge, FUS has the potential to become a new tool for causal mapping of brain function. In addition, studies of the potential of FUS to induce plastic changes in aberrant brain circuits may lead to novel noninvasive approaches to treat neurological and psychiatric disorders.

\section{Acknowledgments}

I thank Dr. Julie Pilitsis for editorial input. I also thank Drs. Daniel Palanker, Julian Brown, Patrick Ye, Martin Prieto, Kamyar Firouzi, Morten Rasmussen, Kim Butts Pauly, Raag Airan, Stephen Baccus, Miriam Goodman, Pierre Khuri-Yakub, and Merritt Maduke for helpful discussions. This work was supported by the NIH grant K99NS100986.

\section{References}

1. Airan RD, Meyer RA, Ellens NP, Rhodes KR, Farahani K, Pomper MG, et al: Noninvasive targeted transcranial neuromodulation via focused ultrasound gated drug release from nanoemulsions. Nano Lett 17:652-659, 2017

2. Barnett SB, Ter Haar GR, Ziskin MC, Rott HD, Duck FA, Maeda K: International recommendations and guidelines for the safe use of diagnostic ultrasound in medicine. Ultrasound Med Biol 26:355-366, 2000

3. Bergey GK, Morrell MJ, Mizrahi EM, Goldman A, KingStephens D, Nair D, et al: Long-term treatment with responsive brain stimulation in adults with refractory partial seizures. Neurology 84:810-817, 2015

4. Bronstein JM, Tagliati M, Alterman RL, Lozano AM, Volkmann J, Stefani A, et al: Deep brain stimulation for Parkinson disease: an expert consensus and review of key issues. Arch Neurol 68:165, 2011

5. Chiken S, Nambu A: Mechanism of deep brain stimulation: inhibition, excitation, or disruption. Neuroscientist 22:313322, 2016

6. Constans C, Deffieux T, Pouget P, Tanter M, Aubry JF: 200$1380-\mathrm{kHz}$ quadrifrequency focused ultrasound transducer for neurostimulation in rodents and primates: transcranial in vitro calibration and numerical study of the influence of skull cavity. IEEE Trans Ultrason Ferroelectr Freq Control 64:717-724, 2017

7. Crowther LJ, Marketos P, Williams P, Melikhov Y, Jiles
DC, Starzewski J: Transcranial magnetic stimulation: improved coil design for deep brain investigation. J Appl Phys 109:07B314, 2011

8. Dallapiazza RF, Timbie KF, Holmberg S, Gatesman J, Lopes $\mathrm{MB}$, Price RJ, et al: Noninvasive neuromodulation and thalamic mapping with low-intensity focused ultrasound. J Neurosurg [epub ahead of of print April 21, 2017. DOI: 10.3171/2016.11.JNS16976]

9. Deffieux T, Younan Y, Wattiez N, Tanter M, Pouget P, Aubry JF: Low-intensity focused ultrasound modulates monkey visuomotor behavior. Curr Biol 23:2430-2433, 2013

10. Duck FA, Baker AC, Starritt HC: Ultrasound in Medicine. Boca Raton: CRC Press, 1998

11. Eames MD, Hananel A, Snell JW, Kassell NF, Aubry JF: Trans-cranial focused ultrasound without hair shaving: feasibility study in an ex vivo cadaver model. J Ther Ultrasound 1:24, 2014

12. Fini M, Tyler WJ: Transcranial focused ultrasound: a new tool for non-invasive neuromodulation. Int Rev Psychiatry 29:168-177, 2017

13. Fry FJ, Ades HW, Fry WJ: Production of reversible changes in the central nervous system by ultrasound. Science 127:8384,1958

14. Gallay MN, Moser D, Rossi F, Pourtehrani P, Magara AE, Kowalski M, et al: Incisionless transcranial MR-guided focused ultrasound in essential tremor: cerebellothalamic tractotomy. J Ther Ultrasound 4:5, 2016

15. Gavrilov LR: Use of focused ultrasound for stimulation of nerve structures. Ultrasonics 22:132-138, 1984

16. Gavrilov LR, Gersuni GV, Ilyinsky OB, Sirotyuk MG, Tsirulnikov EM, Shchekanov EE: The effect of focused ultrasound on the skin and deep nerve structures of man and animal. Prog Brain Res 43:279-292, 1976

17. Gavrilov LR, Tsirulnikov EM, Davies IA: Application of focused ultrasound for the stimulation of neural structures. Ultrasound Med Biol 22:179-192, 1996

18. Ghanouni P, Pauly KB, Elias WJ, Henderson J, Sheehan J, Monteith S, et al: Transcranial MRI-guided focused ultrasound: a review of the technologic and neurologic applications. AJR Am J Roentgenol 205:150-159, 2015

19. Goss SA, Frizzell LA, Dunn F: Ultrasonic absorption and attenuation in mammalian tissues. Ultrasound Med Biol 5:181-186, 1979

20. Grossman N, Bono D, Dedic N, Kodandaramaiah SB, Rudenko A, Suk HJ, et al: Noninvasive deep brain stimulation via temporally interfering electric fields. Cell 169:10291041, 1041.e1-1041.e16, 2017

21. Hameroff S, Trakas M, Duffield C, Annabi E, Gerace MB, Boyle P, et al: Transcranial ultrasound (TUS) effects on mental states: a pilot study. Brain Stimul 6:409-415, 2013

22. Harvey EN: The effect of high frequency sound waves on heart muscle and other irritable tissues. Am J Physiol 91:284-290, 1929

23. Horvath JC, Forte JD, Carter O: Evidence that transcranial direct current stimulation (tDCS) generates little-to-no reliable neurophysiologic effect beyond MEP amplitude modulation in healthy human subjects: A systematic review. Neuropsychologia 66:213-236, 2015

24. Iversen MM, Christensen DA, Parker DL, Holman HA, Chen J, Frerck MJ, et al: Low-intensity ultrasound activates vestibular otolith organs through acoustic radiation force. J Acoust Soc Am 141:4209-4219, 2017

25. Kamimura HA, Wang S, Chen H, Wang Q, Aurup C, Acosta $\mathrm{C}$, et al: Focused ultrasound neuromodulation of cortical and subcortical brain structures using 1.9 MHz. Med Phys 43:5730-5735, 2016

26. Kim H, Chiu A, Lee SD, Fischer K, Yoo SS: Focused ultrasound-mediated non-invasive brain stimulation: examination of sonication parameters. Brain Stimul 7:748-756, 2014 
27. King RL, Brown JR, Newsome WT, Pauly KB: Effective parameters for ultrasound-induced in vivo neurostimulation. Ultrasound Med Biol 39:312-331, 2013

28. Kolbinger HM, Höflich G, Hufnagel A, Müller HJ, Kasper S: Transcranial magnetic stimulation (TMS) in the treatment of major depression-a pilot study. Hum Psychopharmacol 10:305-310, 1995

29. Krasovitski B, Frenkel V, Shoham S, Kimmel E: Intramembrane cavitation as a unifying mechanism for ultrasound-induced bioeffects. Proc Natl Acad Sci U S A 108:3258-3263, 2011

30. Kubanek J, Shi J, Marsh J, Chen D, Deng C, Cui J: Ultrasound modulates ion channel currents. Sci Rep 6:24170, 2016

31. Kubanek J, Shukla P, Das A, Baccus S, Goodman MB: U1trasound elicits behavioral responses through mechanical effects on neurons and ion channels in a simple nervous system. bioRxiv [epub ahead of print], 2017

32. Lee W, Kim HC, Jung Y, Chung YA, Song IU, Lee JH, et al: Transcranial focused ultrasound stimulation of human primary visual cortex. Sci Rep 6:34026, 2016

33. Lee W, Lee SD, Park MY, Foley L, Purcell-Estabrook E, Kim $\mathrm{H}$, et al: Image-guided focused ultrasound-mediated regional brain stimulation in sheep. Ultrasound Med Biol 42:459470,2016

34. Legon W, Sato TF, Opitz A, Mueller J, Barbour A, Williams A, et al: Transcranial focused ultrasound modulates the activity of primary somatosensory cortex in humans. Nat Neurosci 17:322-329, 2014

35. Li GF, Zhao HX, Zhou H, Yan F, Wang JY, Xu CX, et al: Improved anatomical specificity of non-invasive neurostimulation by high frequency $(5 \mathrm{MHz})$ ultrasound. Sci Rep 6:24738, 2016

36. Loo CK, Mitchell PB: A review of the efficacy of transcranial magnetic stimulation (TMS) treatment for depression, and current and future strategies to optimize efficacy. J Affect Disord 88:255-267, 2005

37. Maeda F, Keenan JP, Tormos JM, Topka H, Pascual-Leone A: Modulation of corticospinal excitability by repetitive transcranial magnetic stimulation. Clin Neurophysiol 111:800-805, 2000

38. Mehić E, Xu JM, Caler CJ, Coulson NK, Moritz CT, Mourad PD: Increased anatomical specificity of neuromodulation via modulated focused ultrasound. PLoS One 9:e86939, 2014

39. Menz MD, Oralkan O, Khuri-Yakub PT, Baccus SA: Precise neural stimulation in the retina using focused ultrasound. J Neurosci 33:4550-4560, 2013

40. Menz MD, Ye P, Khuri-Yakub P, Baccus S: Physical mechanisms of ultrasonic neurostimulation in the in vitro retina, presented at Neuroscience 2016, San Diego, November 12-16, 2016 (Abstract) (http://www.abstractsonline.com/ pp8/\#!/4071/presentation/9381) [Accessed December 13, 2017]

41. Naor O, Krupa S, Shoham S: Ultrasonic neuromodulation. J Neural Eng 13:031003, 2016

42. Nightingale KR, Church CC, Harris G, Wear KA, Bailey MR, Carson PL, et al: Conditionally increased acoustic pressures in nonfetal diagnostic ultrasound examinations without contrast agents: a preliminary assessment. J Ultrasound Med 34:1-41, 2015

43. Nitsche MA, Cohen LG, Wassermann EM, Priori A, Lang N, Antal A, et al: Transcranial direct current stimulation: state of the art 2008. Brain Stimul 1:206-223, 2008

44. Parihar R, Alterman R, Papavassiliou E, Tarsy D, Shih LC: Comparison of Vim and STN DBS for parkinsonian resting and postural/action tremor. Tremor Other Hyperkinet Mov (N Y) 5:321, 2015
45. Pascual-Leone A, Valls-Solé J, Wassermann EM, Hallett M: Responses to rapid-rate transcranial magnetic stimulation of the human motor cortex. Brain 117:847-858, 1994

46. Perlmutter JS, Mink JW: Deep brain stimulation. Annu Rev Neurosci 29:229-257, 2006

47. Plaksin M, Kimmel E, Shoham S: Cell-type-selective effects of intramembrane cavitation as a unifying theoretical framework for ultrasonic neuromodulation. eNeuro 3:e0136, 2016

48. Plaksin M, Shoham S, Kimmel E: Intramembrane cavitation as a predictive bio-piezoelectric mechanism for ultrasonic brain stimulation. Phys Rev X 4:011004, 2014

49. Prieto ML, Firouzi K, Khuri-Yakub BT, Maduke M: Mechanical activation of Piezol but not Nav1.2 channels by ultrasound. bioRxiv [epub ahead of print], 2017

50. Prieto ML, Oralkan Ö, Khuri-Yakub BT, Maduke MC: Dynamic response of model lipid membranes to ultrasonic radiation force. PLoS One 8: 77115,2013

51. Rohr KR, Rooney JA: Effect of ultrasound on a bilayer lipid membrane. Biophys J 23:33-40, 1978

52. Sarvazyan AP, Rudenko OV, Nyborg WL: Biomedical applications of radiation force of ultrasound: historical roots and physical basis. Ultrasound Med Biol 36:1379-1394, 2010

53. Syeda R, Florendo MN, Cox CD, Kefauver JM, Santos JS, Martinac B, et al: Piezol channels are inherently mechanosensitive. Cell Reports 17:1739-1746, 2016

54. Trahey GE, Palmeri ML, Bentley RC, Nightingale KR: Acoustic radiation force impulse imaging of the mechanical properties of arteries: in vivo and ex vivo results. Ultrasound Med Biol 30:1163-1171, 2004

55. Tufail Y, Matyushov A, Baldwin N, Tauchmann ML, Georges J, Yoshihiro A, et al: Transcranial pulsed ultrasound stimulates intact brain circuits. Neuron 66:681-694, 2010

56. Tufail Y, Yoshihiro A, Pati S, Li MM, Tyler WJ: Ultrasonic neuromodulation by brain stimulation with transcranial ultrasound. Nat Protoc 6:1453-1470, 2011

57. Tyler WJ: Noninvasive neuromodulation with ultrasound? A continuum mechanics hypothesis. Neuroscientist 17:25-36, 2011

58. Tyler WJ, Tufail Y, Finsterwald M, Tauchmann ML, Olson EJ, Majestic C: Remote excitation of neuronal circuits using lowintensity, low-frequency ultrasound. PLoS One 3:e3511, 2008

59. Velling VA, Shklyaruk SP: Modulation of the functional state of the brain with the aid of focused ultrasonic action. Neurosci Behav Physiol 18:369-375, 1988

60. Wattiez N, Constans C, Deffieux T, Daye PM, Tanter M, Aubry JF, et al: Transcranial ultrasonic stimulation modulates single-neuron discharge in macaques performing an antisaccade task. Brain Stimul 10:1024-1031, 2017

61. Wu J, Lewis AH, Grandl J: Touch, tension, and transduction - the function and regulation of piezo ion channels. Trends Biochem Sci 42:57-71, 2017

62. Ye PP, Brown JR, Pauly KB: Frequency dependence of ultrasound neurostimulation in the mouse brain. Ultrasound Med Biol 42:1512-1530, 2016

\section{Disclosures}

The author reports no conflict of interest concerning the materials or methods used in this study or the findings specified in this paper.

\section{Correspondence}

Jan Kubanek: Stanford University School of Medicine, Stanford, CA.kubanek@stanford.edu. 